\title{
THE CONSTRUCTION OF DESIGN SCIENCE KNOWLEDGE GRAPHS - BASED ON NATIONAL NATURAL SCIENCE FOUNDATION OF CHINA
}

\author{
Xu, Jiang (1); Sun, Gang (1); Ou, Xifan (1); Xu, Jingyu (1); Lu, Han (1); Su, Pujie (1); Wei, Qiushi \\ (1); Ding, Man (2) \\ 1: Tongji University; 2: Hebei University of Technology
}

\begin{abstract}
In the era of knowledge networking, the structure and production mode of knowledge are constantly changing. This article creatively introduces the knowledge mapping method in design research, and based on the perspective of the National Natural Science Foundation of China (NSFC) to compile literature, uses word frequency analysis, co-word analysis, and citation analysis to construct knowledge graphs of design science. This study graphically shows the distribution and flow law of knowledge within design discipline and probes into the research frontier and evolution trend of Chinese design science.
\end{abstract}

Keywords: Design theory, Knowledge graphy, Research methodologies and methods, Design methodology, NSFC

\section{Contact:}

Sun, Gang

Tongji University

College of Design and Innovation

China, People's Republic of

1810839768@qq.com

Cite this article: Xu, J., Sun, G., Ou, X., Xu, J., Lu, H., Su, P., Wei, Q., Ding, M. (2019) 'The Construction of Design Science Knowledge Graphs - Based on National Natural Science Foundation of China', in Proceedings of the 22nd International Conference on Engineering Design (ICED19), Delft, The Netherlands, 5-8 August 2019. DOI:10.1017/ dsi.2019.267 


\section{INTRODUCTION}

The pattern of knowledge production and the structure of scientific knowledge are constantly changing in today's society, and the scientific paradigm of the way of seeking knowledge has been rethought and developed. The voice that breaks the boundaries of overly subdivided and isolated disciplines, and that increasingly complex research topics in an interdisciplinary and integrated approach has surpassed ever before (Guo Yong, 2011). Design science itself is an open system that is comprehensive, crosscutting, and contingent. It requires the support and linkage of many related disciplines and systems, and must constantly expand and transform itself in the intercrossing and integration of disciplines. Simon believes that "designed science" is a third type of knowledge system independent of science and technology (Cross, 2007). In design research, the most important thing is how to find the most important effective information in the interdisciplinary design system and form a theoretical review and systematic review of design science.

The traditional research methods of design mainly rely on personal subjective experience and simple statistics, and neglect the similarity and inheritance between the literature information in the discipline system. The scientific knowledge graph is based on scientific knowledge, and processes vast amounts of literature information through methods such as data mining, information processing, knowledge measurement, and graphic rendering to objectively and accurately display the development process of scientific knowledge, structure relations and evolution relationships (Liu Zeyuan, 2008 and Garfield, 1964). It is both a visualized knowledge map and a serialized knowledge pedigree, which enables the transformation of the knowledge structure from mathematical expression to graphic expression (Kretschmer, 2004).

This study uniquely introduces the knowledge graph in the research of design disciplines, further studies the development trend of design science from the perspective of the National Natural Science Foundation of China, and comprehensively uses co-word analysis, citation analysis and other methods. It visualizes and analyses the abstract knowledge of design science, graphically and accurately explores the research frontier and evolution of design science, and clarifies the status quo and future development trends of China's design scientific research in the age of knowledge network.

\section{DATA SOURCES}

In order to ensure the reliability of the data and to describe the basic situation and research frontier of Chinese design science at the micro level, firstly, this study collects and collates 366 projects of the National Natural Science Foundation of China which are related to design science from 1997 to 2017. On this basis of the project, restricted by project leader and supporting agency, the documents attached to the fund projects are selected, and then the topics, authors, abstracts, keywords, citations, and other information are derived as initial literature data from Chinese Science Citation Database in Web of Science. Subsequently, the experts screen out documents with strong relevance to design, and then the data format of the literature is collated. Finally, 1338 journal articles closely related to design science are analysed as the collated text data of this study, which are imported into softwares such as Citespace V and VOSviewer for further analysis and processing.

\section{ANALYSIS OF RESEARCH HOTSPOTS AND TREND IN DESIGN SCIENCE}

To study the research frontiers and evolution of design science objectively, accurately, and vividly, this study uses co-word analysis, citation analysis, and other methods of bibliometric to construct knowledge graphs of design sciences comprehensively. First, Contents including the key words and abstracts of literature data are analysed with word frequency analysis and co-word analysis, excavating the co-occurrence relationship between high-frequency words to reflect the knowledge distribution laws of design science, and then the research hotspots and directions of design science are studied with cluster analysis based on co-word network. Second, citation analysis is made on the basis of the reference data. The establishment of citation networks, indirectly, reflects the knowledge flow in the design science through the citation relationship of the literature, so as to study the frontiers and trends of design science intuitively and deeply. Finally, knowledge graphs of design science are constructed completely through the synthesis and comparative analysis of the two above. 


\subsection{Knowledge concepts of design science}

Import the collated text data into VOSviewer and extract out words whose frequency of occurrence isn't lower than three, the heat distribution of high frequency words is obtained and showed in Figure 1. From Figure 1, it can be seen that there are many keywords locating in the high heat area, such as Conceptual Design, Product Design, Human-computer Interaction, Innovation Design, Genetic Algorithm, Industrial Design, Axiomatic Design, Design Structure Matrix, Theory of Inventive Problem Solving, Computeraided Design, Kansei Engineering, Product Innovation, Green Design and so on. It indicates that these concepts are ones with top-ranked frequency in the design domain, and the knowledge distributions of design science often form diverse knowledge groups based on these concepts.



Figure 1. Keywords distribution graph in design science

\subsection{Knowledge distribution and research hotspots in design science}

\subsubsection{Co-word network graph}

The document data is collated into the WOS output format after screening and removing duplication, and then is imported into Citespace V software. The co-word analysis function is used to generate keyword co-occurrence knowledge graphs in the design field (Figure 2). There are 3,315 nodes and 8054 links in the graph. The average silhouette score is 0.9683 . The spectral clustering method (Chaomei, 2006) is used to cluster the network, and six main clustering knowledge groups are shown in the graph clearly and intuitively.

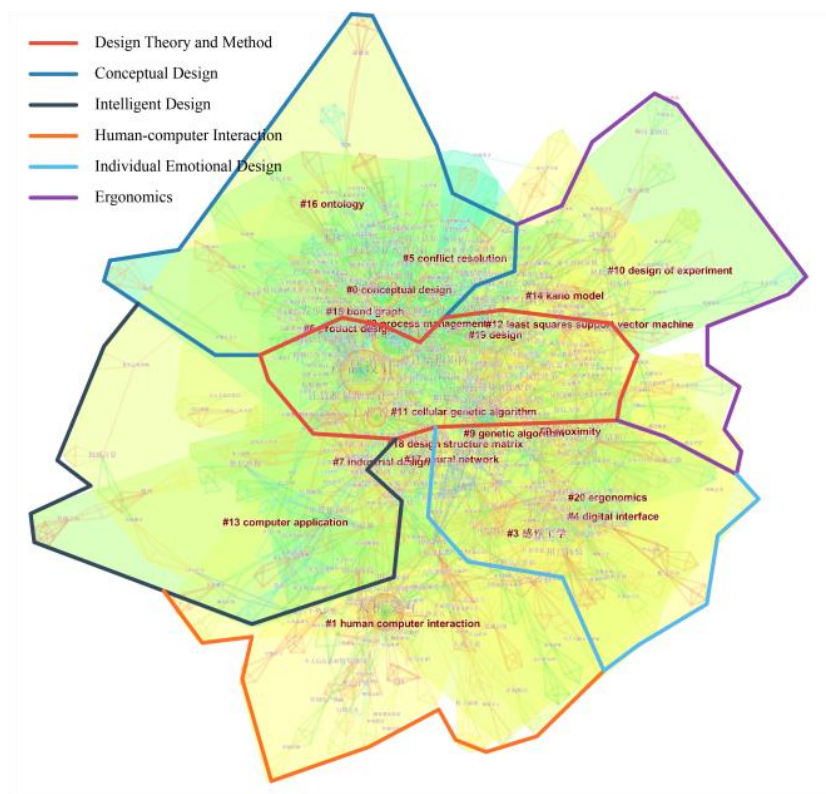

Figure 2. Co-word network graph in design science 
The first major group of knowledge is the field of conceptual design, including Cluster \#0, \#15 and \#16. Cluster \#0 mainly describes the core theories and methods of conceptual design. The keywords include Conceptual Design, Innovative Design, Problem Solving Theory, Process Models, Product Genes, etc. Cluster \#15 and \#16 mainly describe emerging field--design cognition, mainly including Design Cognition, Design Thinking, Feature Extraction, Similarity, Sketch Recognition, Emotion Recognition and other keywords. Conceptual design refers to the entire system workflow, which takes the design requirements and conditions as input and the best solution as the output (Pahl, 1984). It contains a series of design activities from analysing user requirements, defining product functions to forming the overall principle solution and generating concept products. Research in the field of conceptual design includes Conceptual Design Methodology, Product Information Modelling, Reasoning Technology, CAD-Computer Aided Design and many other aspects. From the co-word network graph, it can be seen that the early research on conceptual design focuses on the research of the methodology of conceptual design, including the process model, theory of inventive problem solving and so on. The proposal of product gene provides a new idea for the study of conceptual design. Afterwards, the research on conceptual design focuses on the application of product information modelling and reasoning techniques, mainly on the expression, decomposition, combination of functions and design process solving technology, including evolutionary design, functional structure design, bond diagram, and design evaluation and so on. Later, research mainly focuses on the theory of design cognition, including design thinking, speech recognition, semantic analysis, design thinking, linked tables and other aspects. The conceptual design field focuses on the core theoretical research of design, and provides support for other fields with methodologies and basic theories. Therefore, it is closely related to other fields and is at the core of design theory.

The second major knowledge group belongs to the field of human-computer interaction, focusing on Cluster \#1. It describes the key technologies and principles in the field of human-computer interaction in recent years, mainly including Human-Computer Interaction, User Interface, Eye Tracking, Visualization, Virtual Reality, Gesture Recognition, Gesture Interaction, Situational Awareness and other keywords. Human-computer interaction improves the interaction experience between humans and machines from ergonomics, cognitive science, and other multi-levels subjects that involving crossdomain knowledge. The main directions include multimodal interaction, intelligence, artificial intelligence, virtual reality, augmented reality and so on (Obrenovic, 2004). The human-computer interaction field integrates multiple design methods, uses artificial intelligence algorithms to support knowledge-driven design, and refers extensively to multi-channel information processing, the natural interaction of pen gestures, and interactive sketch tool design. In the co-word graph, the research on human-computer interaction mainly involves interface design, situational awareness, recognition, and pervasive computing. The basic theory of applied design in this field is integrated with computers, network communications and anthropology, forming a relatively independent important knowledge community in the design knowledge graph of design, which profoundly reflects the interdisciplinary characteristics of design science.

The third major knowledge group belongs to the individual emotional design field, including Cluster $\# 2$, \#4 and \#5. Cluster \#2 mainly describes the research hotspots of kansei engineering in recent years, including some keywords, such as Kansei Engineering, Product Modelling, User Experience, Usability and HMI, etc. Cluster \# 4, \#5 mainly describe the application of customized design in product individual innovation design, including Analytic Hierarchy Process, Product Innovation, Quality Function Deployment, Scheme Evaluation and Multi-Attribute Decision-making, etc. The main contents of individual emotional design research consist of two aspects, kansei engineering and customized design. Kansei engineering emphasizes the use of engineering and technique methods to explore the relationship between consumer's perception and product design features (Mitsuo, 1995), and customized design manufactures products according to the personalized requirements of customers to reduce cost of production and enhance advantage in value competition (Tseng, 1996). In the keyword co-occurrence network, the concept of kansei engineering emerges late (2006), whose studies mainly focus on the product image design, mainly involving emotional measurement research and the generation of user perception image, embracing product modelling, user experience, product image, graphical user interface, eye movement experiment and various aspects. Researches in customized design mainly pay attention to mass customization design. After the consumer demand analysis model (Kano model) was put forward in 1984, many scholars gradually concentrate on the theory and methods of product family design and obtain fruitful achievements. During these years, 
modular design, analytic hierarchy process, quality function deployment and grey correlation analysis are mainly applied. Individual emotional design is related tightly to conceptual design, widely using the theories in the field of conceptual design, combined deeply with the techniques employed in ergonomics, forming the important research direction about mining and satisfying user needs.

The fourth major group of knowledge is the field of intelligent design, including four clusters \# 3, \# 7 , \# 8 and \# 10. Cluster \# 3 mainly describes the methods and models applied in intelligent design recent years, including Genetic Algorithms, Neural Networks, Morphological Design, Artificial Intelligence and other keywords; Cluster \# 7 mainly describes the extraction and expression of design knowledge by intelligent design, the keywords include Knowledge Acquisition, Rough set, Collaborative Design, Knowledge Base, Knowledge Representation, Knowledge Engineering, etc. Cluster \# 8 and \# 10 mainly describe computer-aided design and data mining technologies, the keywords include Computer Aided Industrial Design, Knowledge Flow, Knowledge Reuse, Cloud Manufacturing, Data Mining, Cloud Innovation and Cluster Analysis, etc. The research on intelligent design mainly focuses on two aspects: design knowledge and evolutionary design. The development of artificial intelligence and computer technology has promoted the research of knowledge-based design, the research of design knowledge modelling and knowledge reuse, and the inspiration of product examples and patent knowledge, and effectively guided the rapid innovation of products (Aken, 2005). The evolutionary design is based on the biological evolutionary thinking, the use of evolutionary algorithms, especially the combination of genetic algorithm optimization and global search capabilities, the design object in the bottom of the demand for an adaptive design. In the common word spectrum, the research on design knowledge mainly includes knowledge discovery, knowledge acquisition, knowledge representation, knowledge base establishment and knowledge reuse, including the concrete application of rough sets, simulated annealing, case inference and other algorithms. Research on evolutionary design mainly includes the expression and evolution of product gene model, including the application of genetic algorithms, neural networks, morphological design, artificial intelligence and other technologies. The main reason for the connection with design knowledge is conceptual design. Both of them are concerned with the expression of knowledge and concept reasoning. The main correlativity with genetic algorithm is mainly in the area of perceptual engineering.

The fifth major group of knowledge belongs to the field of design theory and method. The clusters are mainly \# 6, \# 11 and \# 12. Cluster \# 6 mainly describes the application of green design method in large-scale product configuration design. Keywords include Green Design, Mass Customization, Multi-objective Optimization, Configuration Design, Product Family, Product Configuration, Product Platform. Cluster \# 11 describes the main theories and methods of optimization design. Keywords include Optimization Design, Experimental design, Response Surface Methodology, Light Weighting, Multidisciplinary Design Optimization, Response Surface Methodology and Sensitivity Analysis. The main description of Cluster \# 12 includes some basic design concepts related to concept design, including Axiomatic Design, TRIZ, Parameterization, Product Innovation Design, Coupled Design and so on. Design theory and method as a scientific approach to achieve design innovation, is mainly divided into two categories: one is the system design theory and methods, including axiom design theory, universal design, quality and function configuration methods, etc. The design is seen as a system composed of a number of independent elements. According to the hierarchical relationships among the elements, organically combining all the design elements, the required tasks for the design system can be achieved; the other category is the specific design theory and method, mainly including optimization design, reliability design, dynamic design, intelligent design, etc. Such methods often have a significant direct impact on design quality and product quality. The field of design theory and method is a summary of the main theoretical methods in other fields. It is roughly located at the centre of the design knowledge graph. It is indirectly related to other disciplines through the specific research fields in the periphery, and achieves the internalization and integration of other disciplines.

The sixth major knowledge group belongs to the field of ergonomics, focusing on Cluster \#9, \#13 and \#14. There are many fields involved in ergonomics. The three main clusters include Support Vector Machines, Eye Tracking, Man-machines Interface, Layout Optimization, Ergonomics, Formalization, User Needs, Comfort, Reverse Engineering, Modal Analysis, and other keywords, mainly describing the widely use of eye movement technology, computer aided technology, and formal technology in the field of ergonomics. It can be seen that ergonomics regards people as the starting point of product design, and requires the appearance, colour, and performance of the product to be concentrated on the physiological and psychological characteristics of the person and to satisfy people's needs and 
capabilities as much as possible. The basic knowledge of ergonomics is mainly coming from engineering psychology, anthropometry, technical aesthetics and so on.

\subsubsection{Analysis of burst terms}

By using Citespace V to conduct a sudden monitoring of the imported literature data, the concept of knowledge with a significant change of word frequency in the literature can be discovered. These high-frequency bursts of knowledge often represent the frontiers and hotspots of the current researches, and reveal the sensitivity, prominence, and dynamic advantages of new research themes with interdisciplinary. Through intensive study of relevant literature of emergence year of sudden word, the turning points in the development of various knowledge areas can be further understood in design science, which is helpful for understanding the development of design science.

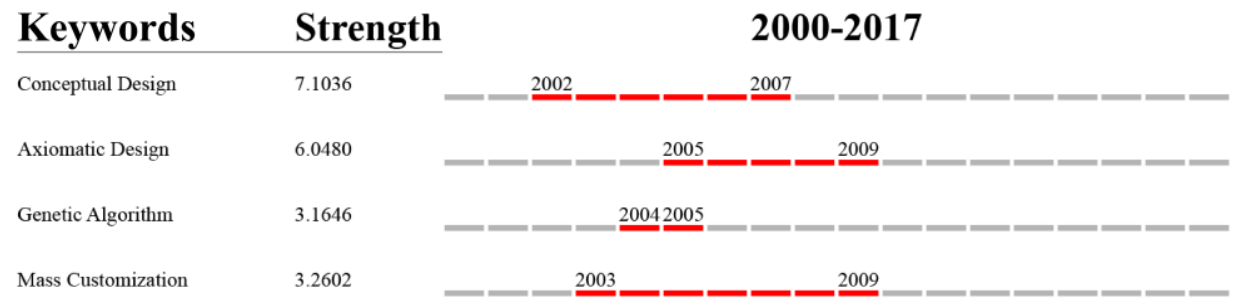

Figure 3. Burst terms of design science

Figure 3 shows the key burst terms of design science. The word "Conceptual Design" increases from 2002 to 2007 as a research hotspot (Figure 4). During this period, He Bin (2007) proposed the share strategies and construction methods of distributed conceptual design knowledge resource to help designers find appropriate principle solution knowledge for conceptual design. Feng Peien (2002, 2004) proposed the concept of "product gene" and its working mechanisms of "transcription", "translation", "copy" and "reverse transcription", established a new methodology of concept design based on the inheritance and reorganization of product genes, and constructed a knowledge base for energy products, which is obviously helpful for product principle innovation in conceptual design. These theoretical methods have greatly enriched the intension of conceptual design and injected new vitality into the development of conceptual design.

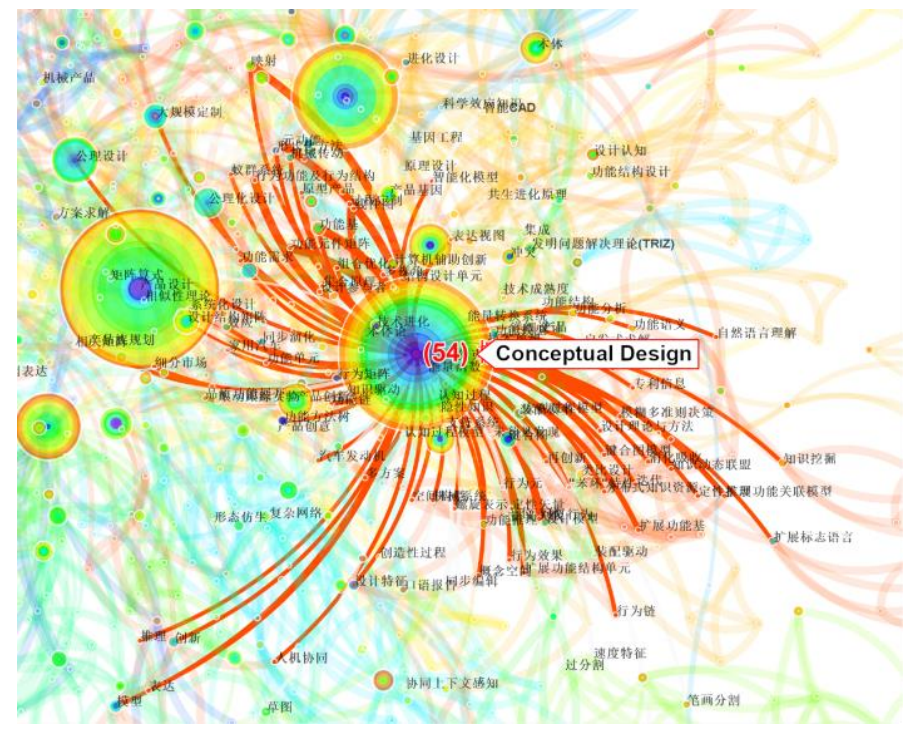

Figure 4. Burstiness of conceptual design

The word "Axiomatic Design" (Figure 5) emerges from 2005 to 2009. During this period, Tang Dunbing (2007) and Wang Xiaoyong (2008) deeply studied the connotation of axiomatic design and proposed the co-evolution mechanism between axiomatized design matrix (ADM) and design structure matrix (DSM), including co-evolution steps and corresponding algorithms. A programmable logic controller (PLC) control system design method based on axiomatic design theory was proposed and the design process model was constructed. 


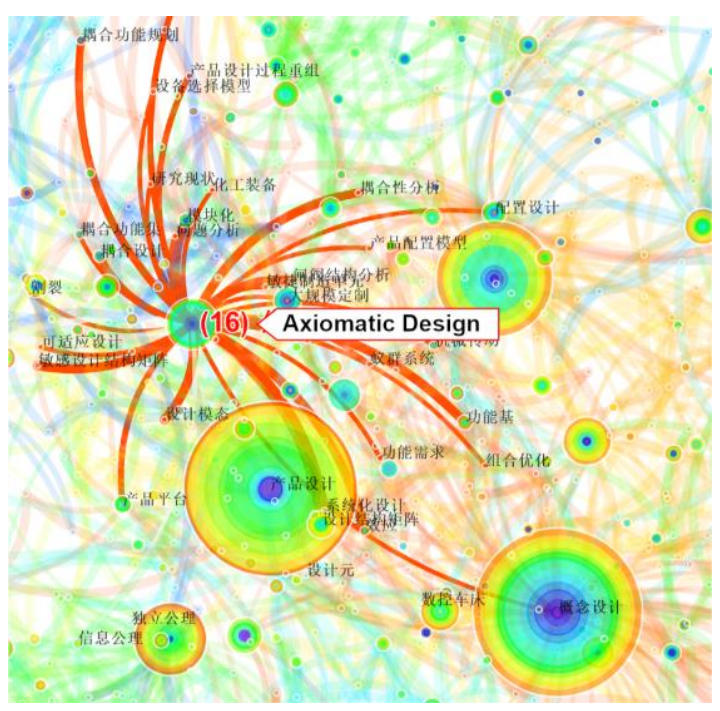

Figure 5. Burstiness of axiomatic design

The word "Genetic Algorithm" (Figure 6) shows a burstiness between 2004 and 2005, in which Liu Xiaojing (2004) adopted genetic algorithms to search solutions to complex surface, and established the proto-system based on the Unigraphics platform. Zhang Gang et al. (2005) referred to the principle of chromosome gene encoding, improved genetic algorithm, proposed a Solution - Vector Coded Genetic Algorithm (SVCGA) to solve layout problems in complex structures of products, and realized the automated spatial planning in three - dimensional layout problems.



Figure 6. Burstiness of genetic algorithm

\subsubsection{Knowledge flow and research frontier in design science}

The citation is the knowledge basis of the scientific text, and the scientific text is the basic unit through which science disseminate and communicate. The process of citation is a process of knowledge evolution based on the predecessors' knowledge, of knowledge selection, inheritance and variation, and of knowledge production, dissemination and application. The citation analysis is a concrete analysis from microcosmic to macro then to microcosmic again, for the process of knowledge flow and the system of knowledge activity (Liang Yongxia et al., 2010). Therefore, this article, in the field of design, uses the method of citation analysis to study the knowledge flow process and discusses the researching trends and frontiers.

As you can see in Figure 7, the citations of the literature in the field of design can be roughly divided into six categories. The early research focuses on two aspects of the invention problem solving, the theory of the invention problem solving and design evaluation, and among which, the key nodes are articles that published by Chiou SJ、Chakrabarti A、Pei-En Feng、Run-Hua Tan、Savransky SD between 1999 and 2002. Mid-term research focuses on the field of axiomatic design, and Suh NP, Su JCY's articles are representative. Later studies mainly focused on the field of emotional design. The representative scholars include Jiang Xu, Xiaojian Liu, Jianning Su, Ye Fu etc. Research in the field of creative design has continued since 2005, and the key nodes are articles published by Guozhong Cao, Yushuai Sun, Howard TJ, Guanglin Ma, RunHua Tan. 


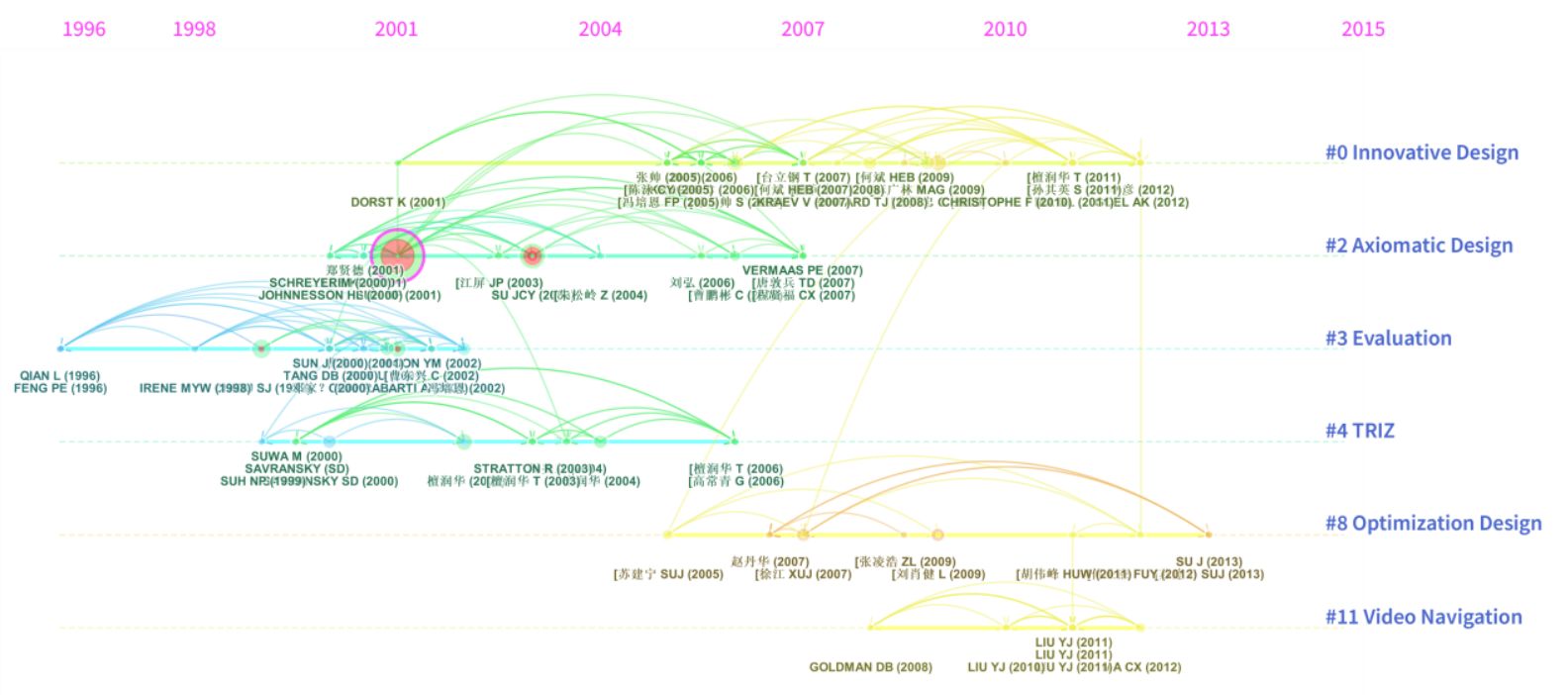

Figure 7. Timeline graph of citation in design science

In the citations graph of design field, those key nodes with high centricity are ground-breaking nodes in certain field, and they represent a relatively stable knowledge base in this field. References to these ground-breaking nodes often reflect the process within this discipline of knowledge collection, dissemination, evolution, and creation of value-added. Therefore, in order to study the nature of the discipline frontier, to clarify the structure of the subject knowledge, and to define and predict the frontier and dynamic trend of research, this paper will deeply search and analyse the ground-breaking nodes in the citation graph (Figure 7).

The ground-breaking literature in design field is the book "Axiomatic Design: Advances and Applications", by Suh NP in 2001. This book introduces in detail the progress and application of axiomatic design, including the basic principles of axiomatic design. Also, it provides principles and practical knowledge for improving axiomatic design, and establishes a reasonable framework for the systematic and scientific basis of design (Suh, 2001). Another ground-breaking literature is the article "A structured approach to measuring functional dependency and sequencing of coupled tasks in engineering design", which was published in "Computers \& Industrial Engineering" by LH Su in 2003. In this article, axiomatic design is used in the design process that multi-factor interacts with each other and segmentation algorithm is adopted to rearrange design matrices. In consequence, the structure and sequence of the design process are revealed, which provides a systematic guidance for engineering design (Su et al., 2003).

The ground-breaking literature in conceptual design is the article "Automated conceptual design of mechanisms" by S.J. Chiou, published in "Mechanism \& Machine Theory" in 1999. This article is a breakthrough in the computer-aided automatic generation of conceptual designs. The author constructs the matrix of finite element, which abstractly expresses scheme representation and component reasoning on different levels to produce a replaceable conceptual design. It turns out the automated generation of the conceptual design is successfully achieved through the computer program (Chiou and Kota, 1999). The foundational literature in individual emotional design is the article "Kansei engineering as a powerful consumer-oriented technology for product development" published by Nagamachi M on "Applied Ergonomics" in 2002. In this paper, the author systematically summarizes the application process of various industries in the 30 years after the appearance of Kansei Engineering and achieves meticulous classification of Kansei Engineering, Kansei Information System, Kansei Engineering System, Hybrid kansei Engineering System, kansei Engineering Model, Virtual kansei Engineering and Collaboration Kansei Engineering System (Nagamachi, 2002).

The article "Sorting algorithm of customer requirements in quality function deployment" written by $\mathrm{Li}$ Yanlai (2007) is an essential article in the custom-design field, published by "Computer Integrated Manufacturing Systems". This paper focuses on the sorting issue of customer requirements based on rough sets in the development of quality functions. The author confirms the importance of customer demands by an essential formula to describe the customer demands competition, which is made by the multi-attribute decision-making method of the rough set in the Incomplete Information System and the 
principle of KANO model. The ground-breaking literature on human-computer interaction design is "Research on pen-based interaction information model" published by Li Jie et al. (2005) in 2005. In this paper, the authors establish orthogonal interaction information architecture coordinate model from the perspectives of design participants and interactive information management. They describe the model from the perspectives of problem knowledge domain, interaction task, rule set to the contextlinked in detail, and have given the implementation based on XML. The fundamental literature in intelligent design was published in 2006 by Von-Wun Soo et al. In the paper, a collaborative multiagent platform based on patent document analysis and ontology are proposed to make a contribution to the innovation process. Another fundamental paper is co-authored by Zhang Hui, Qiu Qingying, Feng Peien and $\mathrm{Wu}$ Jianwei in Computer Integrated Manufacturing System. It proposes a new method to facilitate the efficient acquisition of a patent related to heterogeneous products and a process model is developed to stimulate the design inspiration by means of patents of heterogeneous products.

It can be seen that the conceptual design, design theories and methods provide the core ideas for the development of design science. The axiomatic design theory is an important theoretical basis of the field of design. In all fields of design, the theories and methods of various disciplines are extensively integrated to achieve the orderly inheritance and evolution of internal knowledge in design and the integration, reorganization, creation and appreciation of interdisciplinary knowledge, which reflects the interdisciplinary and innovation of design science.

\section{COCLUSION}

This study creatively introduces the knowledge mapping method into design research, and collects the literature from a perspective of the National Natural Science Foundation of China, applying several biblio-metrological methods to construct knowledge graphs of design science, such as word frequency analysis, co-word analysis, and citation analysis. The main contributions are as follows:

1. Making lots of efforts to obtain and analyse a large and effective project and literature dataset. Since our data sources are objective and obtained from the open access to the online data platform and expert analysis, they are representative and authoritative.

2. Based on the research in cross-design science, this study puts forward a method, which clarifies the macroscopic and microcosmic discipline structure, evolution law and development trend by data visualization.

3. This study may help to put forward new research view and paradigm for the development of interdisciplinary theory, and even to establish the meta research system of design science on the basis of knowledge graphs, and to develop new methods and theories for future design scientific research and design doctor education.

\section{REFERENCES}

Aken, JEV. (2005), "Valid knowledge for the professional design of large and complex design processes", Design Studies, Vol. 26 No. 4, pp. 379-404.

Chaomei, C. (2006), "CiteSpace II: Detecting and visualizing emerging trends and transient patterns in scientific literature", Journal of the American Society for Information Science and Technology, Vol. 57 No. 3, pp. 359-377.

Chiou, S.J. and Kota, S. (1999), “Automated conceptual design of mechanisms", Mechanism and Machine Theory, Vol. 34 No. 3, pp. 476-495.

Cross, N. (2007), "Forty years of design research", Design Studies, Vol. 28 No. 1, pp. 1-4.

Feng, P. Chen, Y. Zhang, S. and P. Shuangxia. (2002), "Product gene based conceptual design", Chinese Journal of Mechanical Engineering, Vol. 38 No. 10, pp. 1-6.

Feng, P. Zhu, A. Chen, Y. Zhang, S. and He, B. (2004), "Establishing knowledge base of product genes for conceptual design of energy transforming systems", Journal of Engineering Design, Vol. 11 No. 3, pp. $113-118$.

Garfield, E., Sher, I. and Torpie, R.J. (1964), "The use of citation data in writing the history of science", Philadelphia Institute of Scientific Information, Vol. 86 No. 4.

Guo, Y. (2011), "General review on the methodology of current design research", Landscape Garden, Vol. 2011 No. 2, pp. 68-71.

He, B, Feng, P. and Pan, S. (2007), "Share strategies and usage methods of distributed conceptual design knowledge resource", Journal of Zhejiang University, Vol. 41 No. 8, pp. 1383-1388.

Kretschmer, H. (2004), "Author productivity and geodesic distance in bibliographic co-authorship networks, and visibility on the Web", Scientometrics, Vol. 60 No. 3, pp. 409-420. 
Li, J. Tian, F. and Dai, G. (2005), "Research on Pen-Based Interaction Information Model”, Journal of Software, Vol. 16 No. 1, pp. 50-57.

Li, Y. Tang, J. Pu, Y. and Yao, J. (2007), "Sorting algorithm of customer requirements in quality function deployment", Computer Integrated Manufacturing Systems, Vol. 13 No. 6, pp. 1196-1203.

Liang, Y. Liu, Z and Yang, Z. (2010), "Explore and discussion on knowledge flowing theory of citation analytics", Studies in Science of Science, Vol. 28 No. 5, pp. 668-674.

Liu, X, Yu, S. and Lu, C. (2004), "Study on Ergonomics CAD of Complex Surface Design in Products", Application Research of Computers, Vol. 21 No. 12, pp. 36-38.

Liu, Z. Chen, Y. and Hou, H. (2008), Science Knowledge Atlas: Methods and Applications, People's Publishing House, Beijing.

Mitsuo, N. (1995), "Kansei Engineering: A new ergonomic consumer-oriented technology for product development", International Journal of Industrial Ergonomics, Vol. 15 No. 1, pp. 3-11.

Nagamachi, M. (2002), "Kansei engineering as a powerful consumer-oriented technology for product development", Applied Ergonomics, Vol. 33 No. 3, pp. 89-94.

Obrenovic, Z. and Starcevic D. (2004), “Modeling Multimodal Human-Computer Interaction”, Computer, Vol. 37 No. 9, pp. 65-72.

Pahl, G. and Beitz, W. (1984), Engineering Design, Design Council, London.

$\mathrm{Su}$, C.Y., Chen, S.J. and Lin, L. (2003), "A structured approach to measuring functional dependency and sequencing of coupled tasks in engineering design", Pergamon Press, Inc., Vol. 45 No. 1, pp. 195-214.

Suh, N.P. (2001), “Axiomatic Design: Advances and Applications”, MIT-Pappalardo Series in Mechanical Engineering.

Tang, D. Qian, X. Wang, X. and Lou, P. (2007), “Coevolution mechanism between axiomatic design matrix and design structure matrix”, Computer Integrated Manufacturing Systems, Vol. 13 No. 8, pp. 1465-1469.

Tseng, M.M., Jianxin, J. and Merchant, M.E. (1996), “Design for Mass Customization”, CIRP Annals, Vol. 45 No. 1, pp. 153-156.

Wang, H. and Sun, B. (2005), "Modular product configuration design for customer requirement-driven engineering”, Chinese Journal of Mechanical Engineering, Vol. 41 No. 4, pp. 85-91.

Wang, H. Wang, J. and Sun, B. (2005), "Optimization design of modularized product family based on kernel platform”, Computer Integrated Manufacturing Systems, Vol. 11 No. 2.

Wang, X. Tang, D. Lou, P. and Ma, W. (2008), “Axiomatic-design-based design method of the programmable logic controller control system”, Computer Integrated Manufacturing Systems, Vol. 14 No. 11, pp. 14651469.

Zhang, G., Yin, G. Deng, K. and Cheng, E. (2005), "Modified real - coded genetic algorithm and its application to layout design", Computer Integrated Manufacturing Systems, Vol. 11 No. 10, pp. 1451-1455.

\section{ACKNOWLEDGMENTS}

This research is based upon work supported by the National Science Foundation of China under Grant No.51205059, No.51675382, No.51575158, and No.61303037, the Major Consulting Projects of Chinese Academy of Engineering under Grant No. 2013ZD15, Shanghai Summit Discipline in Design under No. DA17001 and DB18106, Chenguang Plan Project of Shanghai Educational Development Foundation under No.15CG72, Tongji University Graduate Education Reform and Research Project under No. 2018GH27003. 